\title{
Editorial: Policies and Strategies for the Conservation of Metacommunities
}

\author{
Martin Drechsler ${ }^{1 *}$, Heidi J. Albers ${ }^{2}$ and Henrik G. Smith ${ }^{3}$ \\ ${ }^{1}$ Department of Ecological Modelling, Helmholtz Centre for Environmental Research, Leipzig, Germany, ${ }^{2}$ Department of \\ Economics, University of Wyoming, Laramie, WY, United States, ${ }^{3}$ Centre for Environmental and Climate Science, Lund \\ University, Lund, Sweden
}

Keywords: biodiversity, conservation, land use, metacommunity, policy, economic, food web, species interaction

Editorial on the Research Topic

Policies and Strategies for the Conservation of Metacommunities

\section{INTRODUCTION}

To halt the ongoing decline of biodiversity, policies and strategies are needed that reduce, exclude or even reverse harmful influences on ecosystems. Theoretical (modelling) and empirical approaches across various fields of conservation science and policy have been developed as powerful tools to assess the performance of conservation policies. As a result, a large body of literature exists on the impact of policies on single or multiple independent species. However, many approaches ignore complexities of ecological systems, which limits their usefulness for informing policy. A challenge here is how to incorporate the most salient features of the complexity of ecosystems and the multitude of possible ecological responses to the human actions as an integral part of policy analyses. In this Research Topic, we emphasise two interlinked dimensions of this complexity. First, the spatio-temporal heterogeneity of ecosystems means that a single blanket policy will not result in the same outcomes across the landscape or across time; incorporating that heterogeneity into analysis can help prioritise and target actions in ways that leverage the heterogeneity to produce landscape level impacts. Second, species are usually organised in ecological networks or metacommunities with important interspecific interactions (competition, trophic interactions) that may result in complex and sometimes surprising outcomes of policies on community change; incorporating those interactions into policy analyses enables the analyst to capture such indirect effects on the impact on non-target species of specific policies or to assess the trade-offs across species from general policies. Yet, large gaps still exist in our understanding of how conservation policies operate through spatio-temporal heterogeneity and species interactions to generate conservation outcomes in complex systems.

Filling this gap requires the integration of knowledge about conservation policy design and ecological multi-species concepts. These two fields have developed substantially in the past decades but have rarely come into contact with each other.

\section{SPECIAL ISSUE OVERVIEW}

This Research Topic presents eight examples in which the two fields have been integrated. In alphabetical order. 
Albers et al., Conservation Actions in Multi-Species Systems: Species Interactions and Dispersal Costs, analyse how species interactions and species dispersal influence affect the allocation of conservation resources in a sagebrush community in Wyoming.

Berthon et al., Small-scale habitat conditions are more important than site context for influencing pollinator visitation, determine empirically the impact of conservation measures on pollinator presence in vegetation patches in Munich.

Brockmann et al., Space and Species Interactions in Welfare Estimates for Invasive Species Policy, demonstrate the biases in recreational fisher welfare estimates from invasive species policies in Lake Michigan from inappropriately simple ecological assumptions.

Drechsler, On the cost-effective design of agglomeration bonus schemes for the conservation of multiple competing species, explores the impact of payment-induced spatial aggregation of conservation measures on species subject to the competitioncolonisation trade-off.

Lee et al., Socio-ecological outcomes of single-species fisheries management: the case of Yellow Perch in Lake Erie, consider the impacts of tradable fishing quotes on a marine food web in Lake Erie.

Solis-Sosa et al., A Landscape-Level Assessment of Restoration Resource Allocation for the Eastern Monarch Butterfly, determine where milkweed should be planted to maximise conservation effects on the Monarch Butterfly in North and Central America.

Wang et al., Delineating conservation areas for cohabiting species: An example of vernal pond conservation from Fort Stewart in Georgia, USA, explore how the interaction between a tortoise and a frog species affect the cost-effective selection of reserve sites in a military area in the US.

Wilman, Forests: Catastrophes, Resilience and Management, analyses how disturbances and conservation management actions affect the resilience of two marine trophic chains in Tasmania, Australia, and Haida Gwaii, Canada.

All papers deal with the impact of conservation policies and strategies on the composition and abundance of species in multi-species ecosystems, with a particular focus on costeffectiveness in Albers et al., Drechsler, Solis-Sosa et al. and Wang et al., The policies considered in the different studies range from the implementation of harvesting control measures
(Wilman) via conservation planning/allocation of conservation resources (Albers et al.; Berthon et al.; Brockmann et al.; Solis-Sosa et al.; Wang et al.;) to market-based instruments, in particular conservation payments and tradable permits (Drechsler; Lee et al.). Species interactions include consumerresource interaction (Solis-Sosa et al.), commensalism (Wang et al.), mutualism (plant-pollinator) (Berthon et al.), competition (Drechsler), trophic chain (Wilman), and food webs (Albers et al.; Brockmann et al.; Lee et al.). Spatial structure is considered via patch-based (Albers et al.; Berthon et al.; Brockmann et al.; Lee et al.; Solis-Sosa et al.;) or grid-based approaches (Drechsler; Wang et al.). Both terrestrial (Albers et al.; Berthon et al.; Drechsler; Solis-Sosa et al.; Wang et al.) and aquatic ecosystems (Brockmann et al. Lee et al.; Wilman;) are considered.

\section{AUTHOR CONTRIBUTIONS}

All authors listed have made a substantial, direct, and intellectual contribution to the work and approved it for publication.

\section{ACKNOWLEDGMENTS}

We would like to thank the peer reviewers for their valuable contributions to the improvement of the manuscripts published in this Research Topic.

Conflict of Interest: The authors declare that the research was conducted in the absence of any commercial or financial relationships that could be construed as a potential conflict of interest.

Publisher's Note: All claims expressed in this article are solely those of the authors and do not necessarily represent those of their affiliated organizations, or those of the publisher, the editors and the reviewers. Any product that may be evaluated in this article, or claim that may be made by its manufacturer, is not guaranteed or endorsed by the publisher.

Copyright (c) 2021 Drechsler, Albers and Smith. This is an open-access article distributed under the terms of the Creative Commons Attribution License (CC BY). The use, distribution or reproduction in other forums is permitted, provided the original author(s) and the copyright owner(s) are credited and that the original publication in this journal is cited, in accordance with accepted academic practice. No use, distribution or reproduction is permitted which does not comply with these terms. 